Introduction: Anaplastic thyroid carcinoma is a rare, rapidly progressing, highly aggressive thyroid malignancy. Responses to immune checkpoint inhibitors in mismatch repair-deficient/ microsatellite instability-high tumours of other locations have shown promising results, and with the extended approval of the PD-1 receptor inhibitor pembrolizumab by the Food and Drug Administration, also anaplastic thyroid cancer (ATC) requires analysis for microsatellite instability (MSI) status. Material and methods: Systematic research for relevant literature was conducted in different databases. Prevalence, detection methods, and the potential prognostic/predictive value of MSI in view of the available targeted therapies were of special focus. Results: Selected citations revealed the prevalence of MSI in $7.4 \%$, with mutations in the MSH2 gene (33\%) being the most frequent, followed by MSH6 (25\%) and MLH1 (16.7\%) occurring in the following combinations: MLH1MSH2 (8.3\%), MSH2-MSH6 (8.3\%), and MLH3-MSH5 (8.3\%). No mutations in the PMS2 gene were reported. Sixty-six co-mutations in 9 cases were found, with TP53 (88.9\%), NF1 (44.4\%), ATM (33.3\%), and RB1 (33.3\%) being the most frequent. No RAS mutations were noted. Survival ranged between 2.8 and 48 months, and patient age varied between 49 and 84 years. There are insufficient and heterogenous data concerning the predictive or prognostic value of mismatch repair-deficient/ microsatellite instability status.

Conclusions: Tumour molecular profiling is fundamental in ATC for predictive, prognostic, as well as therapeutic reasons, and analysis of MSI status is strongly suggested because a small subgroup show the MSI signature and might profit from recently approved targeted therapies.

Key words: anaplastic thyroid cancer, microsatellite instability, MMR, immunotherapy, targeted therapy.

Contemp Oncol (Pozn) 2021; 25 (3): 213-223 DOI: https://doi.org/10.5114/wo.2021.110052

\section{The prevalence of DNA microsatellite instability in anaplastic thyroid carcinoma - systematic review and discussion of current therapeutic options}

\author{
Maria Linda Rocha ${ }^{1}$, Kurt Werner Schmid², Piotr Czapiewski ${ }^{3,4}$
}

${ }^{1}$ Institute of Pathology Königs Wusterhausen, Königs Wusterhausen, Germany ${ }^{2}$ Institute of Pathology, University Hospital Essen, University of Duisburg-Essen, Essen, Germany

${ }^{3}$ Institute of Pathology, Dessau Medical Centre, Dessau, Germany

${ }^{4}$ Institute of Pathology, Otto-von-Guericke University Magdeburg, Magdeburg, Germany

\section{Introduction}

Anaplastic thyroid cancer (ATC) represents by far the most aggressive type of primary malignant thyroid cancer, arising from transformation of differentiated thyroid carcinoma or de novo and constituting $1-2 \%$ of all thyroid malignancies, but having the greatest clinical impact on cancer-related deaths $[1,2]$. In Europe, the cancer incidences, given as age-standardized rates, are low at $<0.3 / 100,000$ in females and $<0.2 / 100,000$ in males [3]. Findings from an epidemiological study in the United States indicated that the incidence rate from 1973 to 2014 increased by 3.0\% annually [4]. In Europe, however, cancer incidence rates appear lower with an annual percentage change (APC) of $+1.3 \%$ according to a large Dutch study [5], and even decreasing rates in females and males were noted according to a large Danish study [6]. Anaplastic thyroid cancer mostly affects older patients, with the majority being over 60 years old, and it is commonly diagnosed at an advanced stage of disease (by definition stage IV according to the staging system of the American Joint Committee on Cancer) with rapid tumour progression and local or distant metastases in up to $30-40 \%$ of patients $[7,8]$. The diagnosis of ATC requires thorough conventional and immunohistochemical exclusion of differential diagnoses such as well-differentiated thyroid carcinoma (WDTC), poorly differentiated thyroid carcinoma (PDTC), medullary thyroid carcinoma (MTC), angiosarcoma, sarcoma, lymphoma, squamous cell carcinoma, metastasis to the thyroid from solid tumours, and Riedel thyroiditis. The concomitant resistance to radiotherapy and systemic chemotherapy contributes to extremely poor overall survival (median 3-10 months; overall disease-specific mortality rate: 68.4\% at 6 months, $80.7 \%$ at 12 months) despite multimodal aggressive first-line therapeutic approaches [7-10]. With increasing knowledge of the tumour biology, the identification of the underlying genetic pathways, modifications of the transcriptome and proteome and associated immunomodulatory mechanisms of ATC on the one hand, and the emerging role of novel, molecular-based single/multi-targeted therapies on the other hand, a growing number of human early clinical trials can be noted [11, 12] (www.clinicaltrials. gov). In addition, international and national guidelines need to be adapted and brought up to date to evaluate established procedures and to implement evidence-based findings, because the recommendations of the American Thyroid Association date back to 2012, those from the National Comprehensive Cancer Network to 2017, and from the British Thyroid Association to 2014. There are currently no available standardized European or German 
treatment guidelines (German S3-Leitlinie, registration number 031-0560L, is scheduled for 31 December 2021 according to Arbeitsgemeinschaft der Wissenschaftlichen Medizinischen Fachgesellschaften e. V).

In recent years, several studies have confirmed that the molecular profile of ATCs includes a small proportion of tumours that demonstrate acquired mutations in the DNA mismatch repair (MMR) pathway (up to 10-15\%), being linked to impaired or deficient post-replicative DNA repair mechanisms [13-19]. As a functional consequence, errors that occur during replication processes might not be recognized properly and be corrected, leading to a dysregulation of MMR and resulting in length aberrations of short repetitive DNA sequences (called short tandem repeats [STRs] or simple sequence repeats [SSRs]) along the whole genome. This results in microsatellite instability (MSI), which is known to drive carcinogenesis and tumour progression $[14,17,20]$.

Therefore, this data analysis aimed to systematically review and meta-analyse the prevalence of impaired DNA MMR status or MSI in ATC and investigate its potential prognostic/predictive value in view of available targeted therapies, with the intention of ensuring optimal individual patient care.

\section{Material and methods}

Formal consent was not required for this review.

\section{Search strategies}

The search was conducted in different electronic databases (PubMed [MEDLINE], Google Scholar, and ASCO),

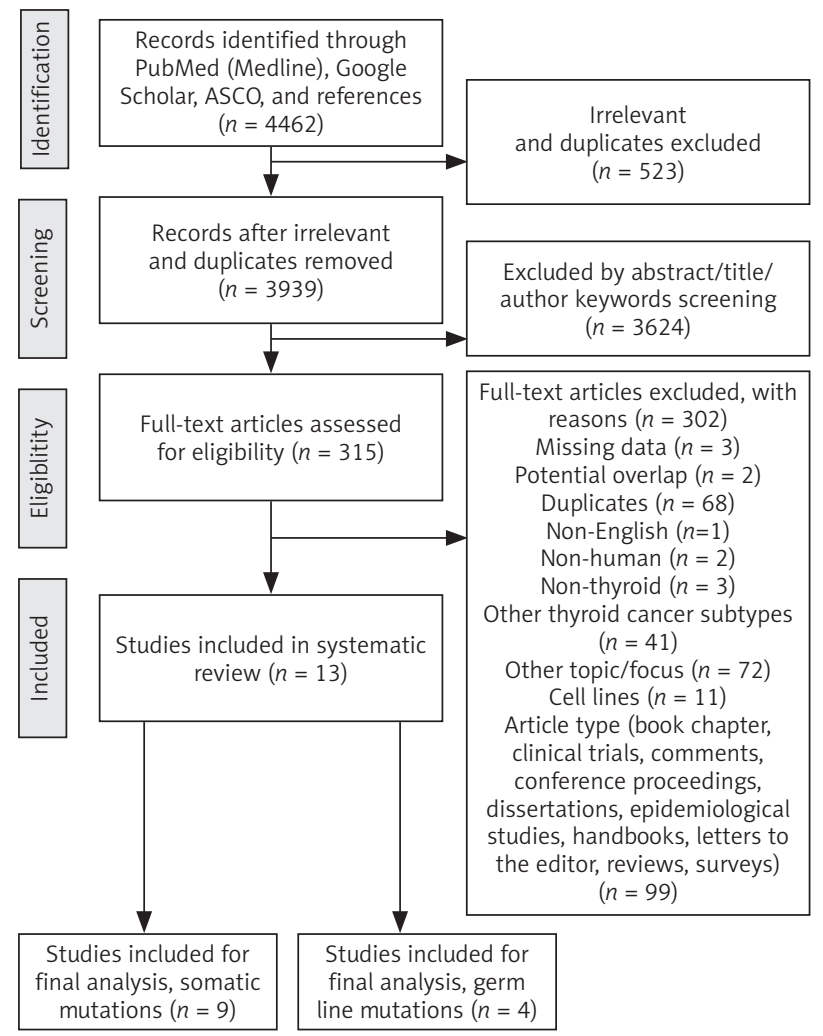

Fig. 1. PRISMA flow diagram visualizing study approach for MMR-d/MS prevalence in anaplastic thyroid cancer (adapted from Moher et al. 2009) and libraries of interest were imported into the Mendeley citation manager. Search terms included "anaplastic thyroid carcinoma", "anaplastic thyroid cancer", "DNA mismatch repair", "mismatch repair deficiency", and "microsatellite instability". Search filters were applied as follows: text availability (abstract and full text), species (human), language (English), and source type (journal). This investigation followed the Preferred Reporting Items for Systematic Review and Meta-analysis (PRISMA) [21].

\section{Critical assessment of selected articles and data acquisition}

Research for appropriate data followed standardized predefined parameters. Title, abstract, and author keywords were screened for all recorded studies, and 2 authors (MLR and PC), each a consultant of pathology, independently reviewed and critically discussed the selected data. The quality if the studies and their potential bias were evaluated. The process of categorization into subgroups and final study selection was performed as shown in the PRISMA flowchart, adapted and modified in accordance with Moher [21] (Fig. 1). Extracted information included: first author, year of publication, total case number, number of included and analysed ATCs, detection methods, and MSI rate. Additional information, such as molecular phenotype, detected mutations in the MMR genes, type of molecular modifications in the MMR genes, frequent co-mutations, prior treatment, and clinical features, was not considered as mandatory, but optional. Studies with potential overlap, an insufficient reference panel, or isolated MMR gene investigation were excluded from the final analysis.

\section{Statistical analysis}

Pooled data were prepared and imported into Excel, Microsoft Office 365. Different observational features (frequencies of MMR gene mutations, associated molecular phenotype, frequencies and types of co-mutations) were analysed as descriptive statistics.

\section{Results}

\section{Results from systematic data research} and meta-analysis

A systematic search approach in different electronic databases initially identified 4462 citations, from which 315 potential full-text articles were finally checked for eligible criteria. Of these, 13 studies qualified for final analysis, 9 of them considering somatic mutations, and 4 of them documenting germ line mutations in the setting of Lynch syndrome (Fig. 1). The inclusion criteria and additional information of the 9 studies without suggested hereditary association are listed in Table 1 and served for metaanalytic evaluation. In total, 175 cases diagnosed with ATC were tested for MSI. Among them, 13 cases (7.4\%) were verified with MSI+ status by different detection methods. For 12 of these cases, the molecular phenotype was given, with 11 cases (91.6\%) presenting with a disproportionally higher tumour mutational burden (TMB; 'hypermutated' phenotype) as a genetic signature (Table 1, Fig. 2) concordant with previous reports in the literature [13-18, 20, 22]. 


\begin{tabular}{|c|c|c|c|c|}
\hline$\ddot{\ddot{\Xi}}$ & 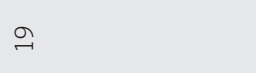 & \pm & 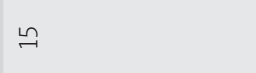 & $\div$ \\
\hline 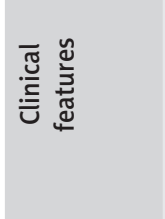 & 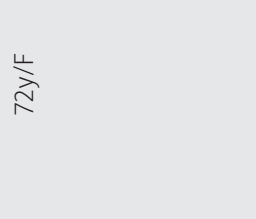 & 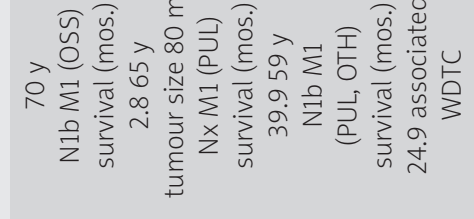 & $\bar{z}$ & 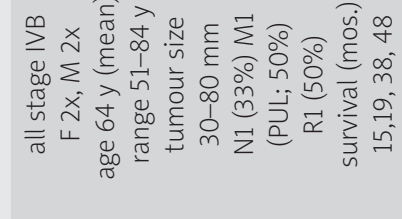 \\
\hline 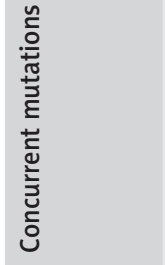 & $\frac{s}{z}$ & 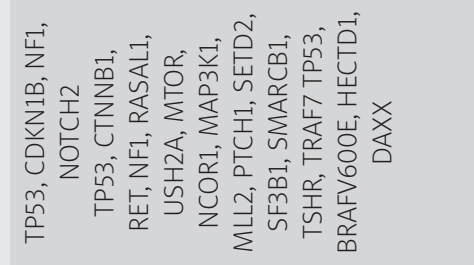 & 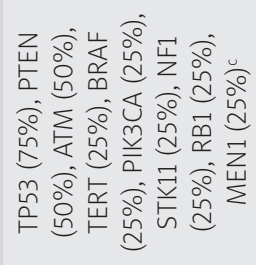 & $\frac{100}{2}$ \\
\hline 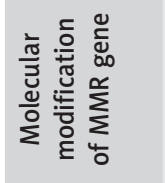 & $\frac{\pi}{z}$ & 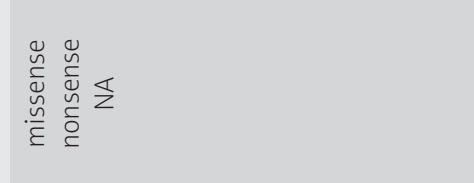 & 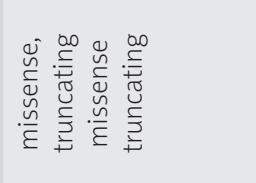 & 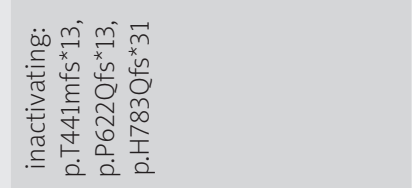 \\
\hline 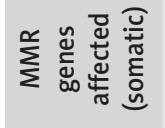 & $\Sigma$ & 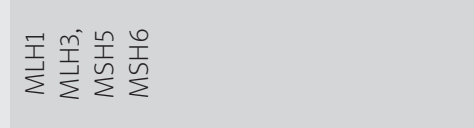 & 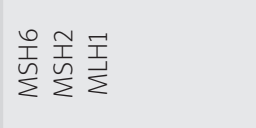 & 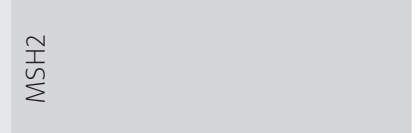 \\
\hline 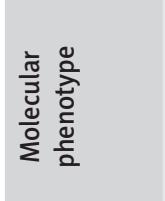 & $\stackrel{s}{z}$ & 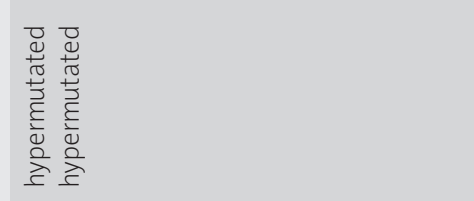 & 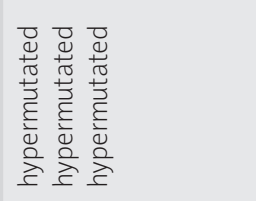 & 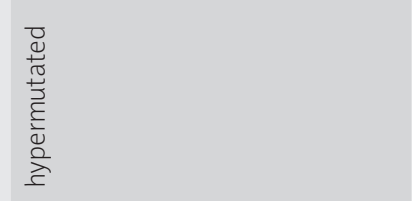 \\
\hline 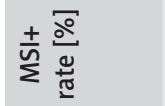 & in & そヂ そ & 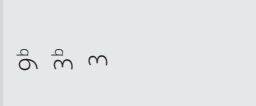 & Oे \\
\hline 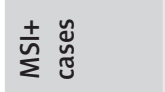 & $\cong$ & $\underset{\Xi}{\Xi}$ & 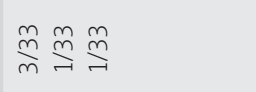 & $\stackrel{\infty}{\sim}$ \\
\hline 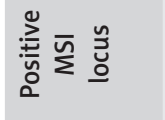 & 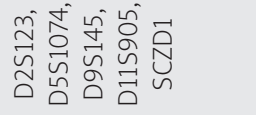 & & $\underline{z}$ & $\frac{\pi}{2}$ \\
\hline 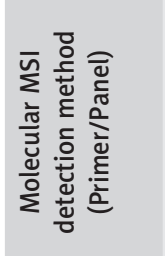 & 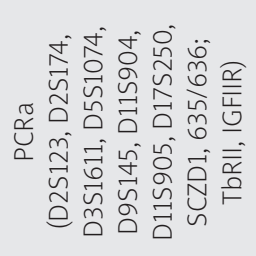 & 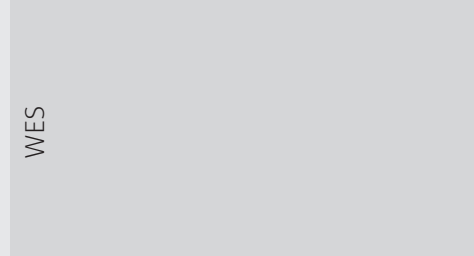 & 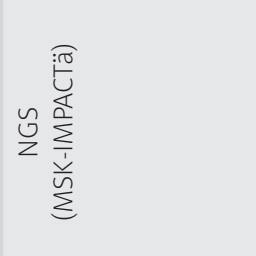 & 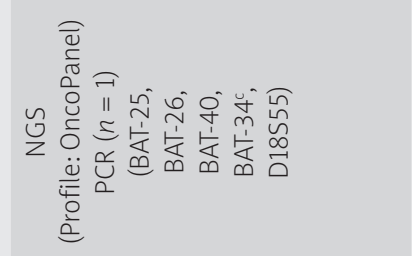 \\
\hline 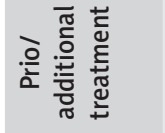 & $\underline{z}$ & 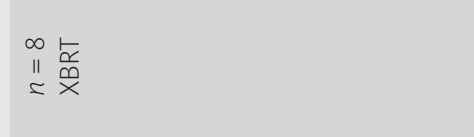 & $\Sigma$ & 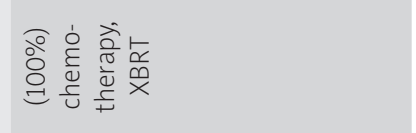 \\
\hline 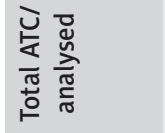 & $\stackrel{\pi}{=}$ & $\underset{\substack{\text { II } \\
=}}{=}$ & $\prod_{\substack{I I \\
=}}$ & $\underset{\substack{\| \\
\simeq}}{=}$ \\
\hline 㰧怘 & $\begin{array}{l}\overrightarrow{\text { In }} \\
\stackrel{\text { II }}{=}\end{array}$ & $\underset{\mathbb{N}}{\stackrel{N}{\Perp}}$ & 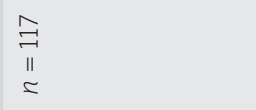 & $\stackrel{\infty}{\stackrel{\infty}{\Perp}}$ \\
\hline ฮ্ & बे & $\stackrel{n}{\stackrel{n}{n}}$ & పั & वे \\
\hline 高 & 空 & 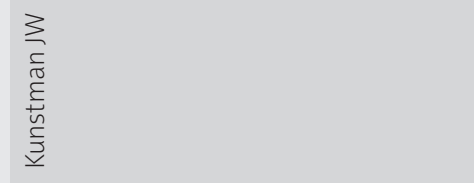 & $\begin{array}{l}\frac{\bar{\sigma}}{\bar{\sigma}} \\
\text { 产 }\end{array}$ & 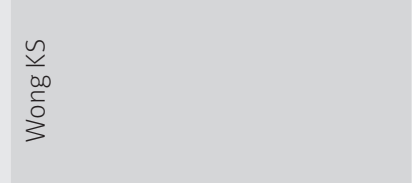 \\
\hline$\stackrel{\circ}{z}$ & - & n & m & $\sigma$ \\
\hline
\end{tabular}




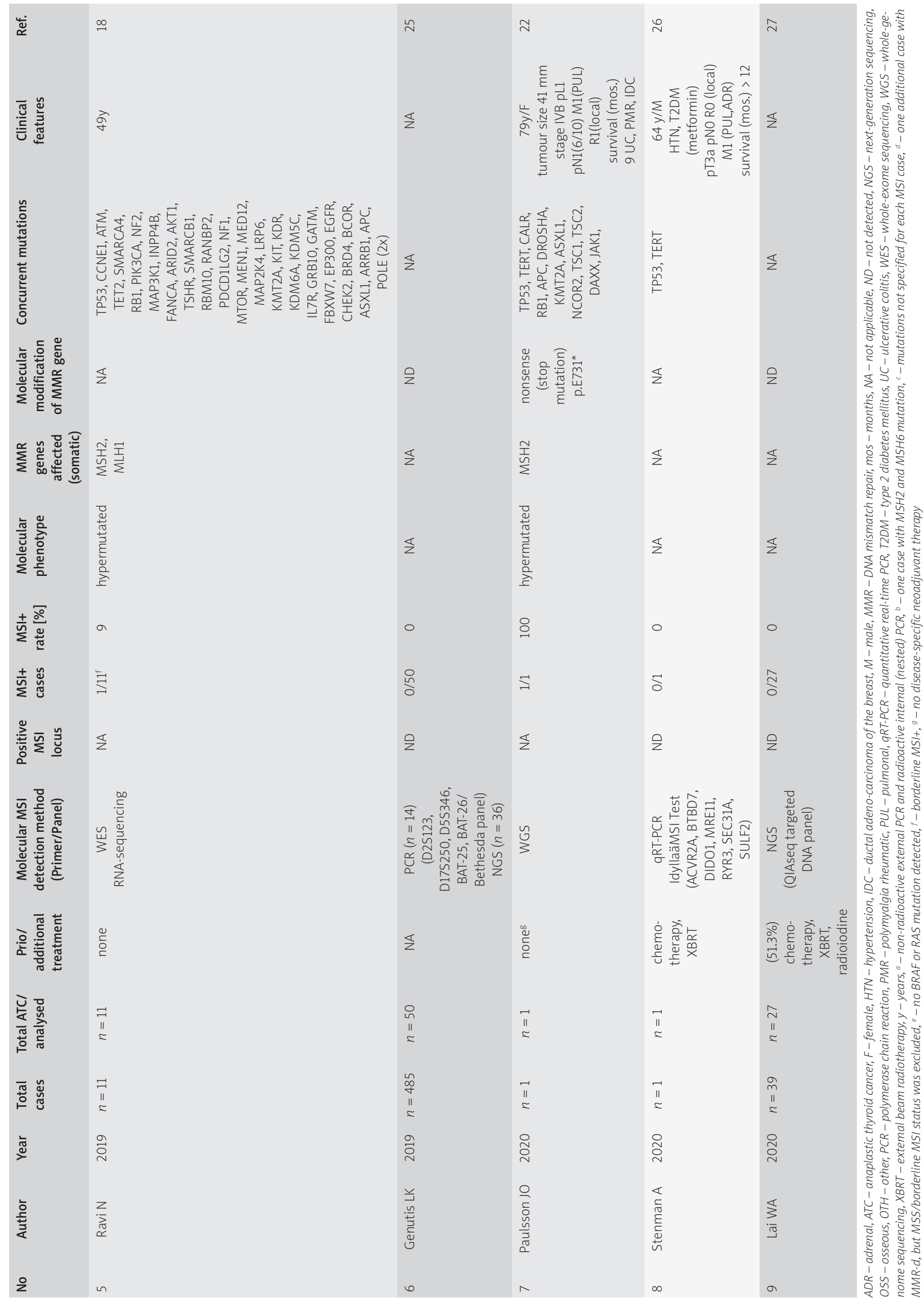


In the landscape of ATC, the affected and documented MMR genes thus far include MutL homologue 1 (MLH1), MutS homologue 2 (MSH2), MutL homologue 3 (MLH3), MutS homologue 5 (MSH5), and MutS homologue 6 (MSH6) (Table 1), with mutations in the MSH2 gene (33\%) being the most frequent, followed by MSH6 (25\%) and MLH1 (16.7\%) (Fig. 2). Mutations in 2 MMR genes occurred in combinations as follows: MLH1-MSH2 (8.3\%), MSH2-MSH6 (8.3\%), and MLH3-MSH5 (8.3\%) (Table 1, Fig. 2). Interestingly, mutations in the gene encoding the MMR endonuclease PMS2 have hitherto not been described, which could be suggestive of low frequencies of PMS2 mutations in general or indicative of more complex underlying mechanisms, implying insufficient detection methods or difficult data interpretation so far. A study conducted on patients with colorectal cancer (CRC) in search for germline mutations in PMS2 (after having found an isolated loss in the immunohistochaemia but preserved MLH1 expression) described significant complications in the detection of mutations due to paralogous genes [23]. Final analysis additionally revealed 66 co-mutations documented in 9 cases, with TP53 (88.9\%), NF1 (44.4\%), ATM (33.3\%), and RB1 (33.3\%) being the most commonly detected (Fig. 3). These were followed by APC, ASXL1, BRAF, DAXX, KMT2A, MAP3K1, MEN1, MTOR, PIK3CA, PTEN, SMARCB1, TERT, and TSHR (each 22.2\%) (Fig. 3). Of note, RAS mutations have not been reported. Survival ranged between 2.8 and 48 months $(n=9)$, and patient age varied between 49 and 84 years (Table 1).

\section{Remarks on included studies}

The study of Wong et al. demonstrated, in a series of 28 ATCs, that MMR-deficient (MMR-d) and MMR-proficient (MMR-p; intact MMR protein expression) tumours did not show any dissimilarities in clinicopathological parameters such as macroscopic tumour size, extrathyroidal tumour expansion, positive nodal status, resection margin status, or histological pattern [16, 24]. Interestingly, however, 3 cases showed a prominent perilesional inflammatory infiltration. The prevalence of MMR-d status was given with 4/28 ATCs (14\%), although 1 case could not convincingly be verified as MSI lacking mutations in the DNA MMR genes MLH1, MSH2, MSH6, PMS2, and EPCAM. It was also reported by Wong that all cases were devoid of WDTC, only partially presenting with foci of PDTC $[16,24]$. Nevertheless,

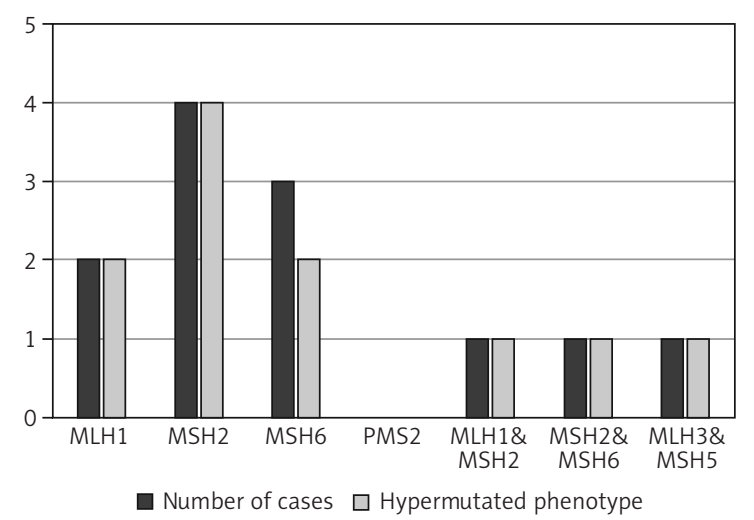

Fig. 2. Distribution of mutated MMR genes within the MSI-positive ATC cohort of the finally included studies (available for $n=12$ ) and the proportion of hypermutated phenotype status (available for $n=11$ )

MMR-d cases tended to have significantly better overall survival rates (1 case reaching 48 months) and presented with fewer metastases at the time of diagnosis [16]. The study of Ravi also included 1 case with borderline MSI status [18]. For only 1 case, an associated well-differentiated tumour component was reported [14]. Three of the studies did not detect MSI cases [25-27].

\section{Comment on excluded studies}

Potential overlap was identified in 2 studies [13, 17], which were therefore excluded. A panel of only 3 microsatellite markers with isolated focus on MSH2 was also not considered in the final analysis [28]. The study by Dong et al. was not taken into account either, because the discovered MSH6 mutation in 1/5 ATCS was only detected in the well-differentiated tumour component (PTC) [29]. Potential risks of bias were identified during data extraction and include neoadjuvant therapy, differences in applied molecular testing methods, and possible ATCs as a minor component only. The latter fact was not specified by any study included.

\section{Original case reports with anaplastic thyroid cancer manifestation within the spectrum of Lynch syndrome}

On extremely rare occasions, ATC (and PTC) may manifest as a non-typical index tumour within the spectrum of Lynch syndrome (LS), an autosomal dominant inherited cancer syndrome, formerly known as hereditary non-polyposis

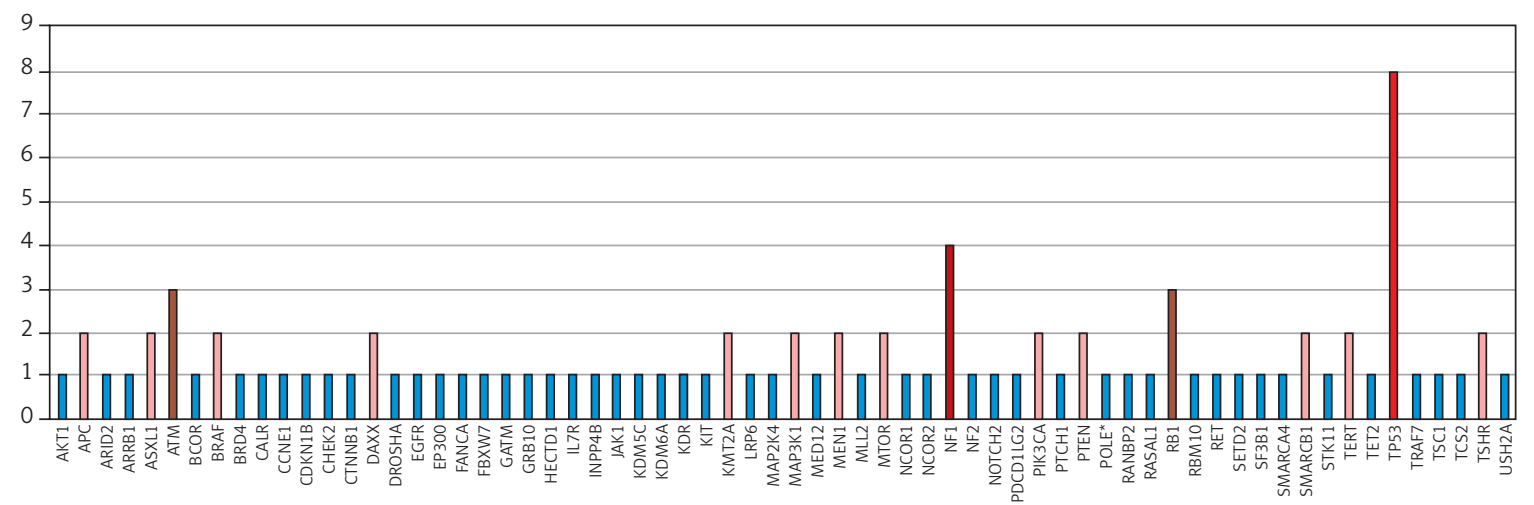

Fig. 3. Distribution of co-mutations within the MSI-positive ATC cohort of the finally included studies (available for $n=9$ ) 


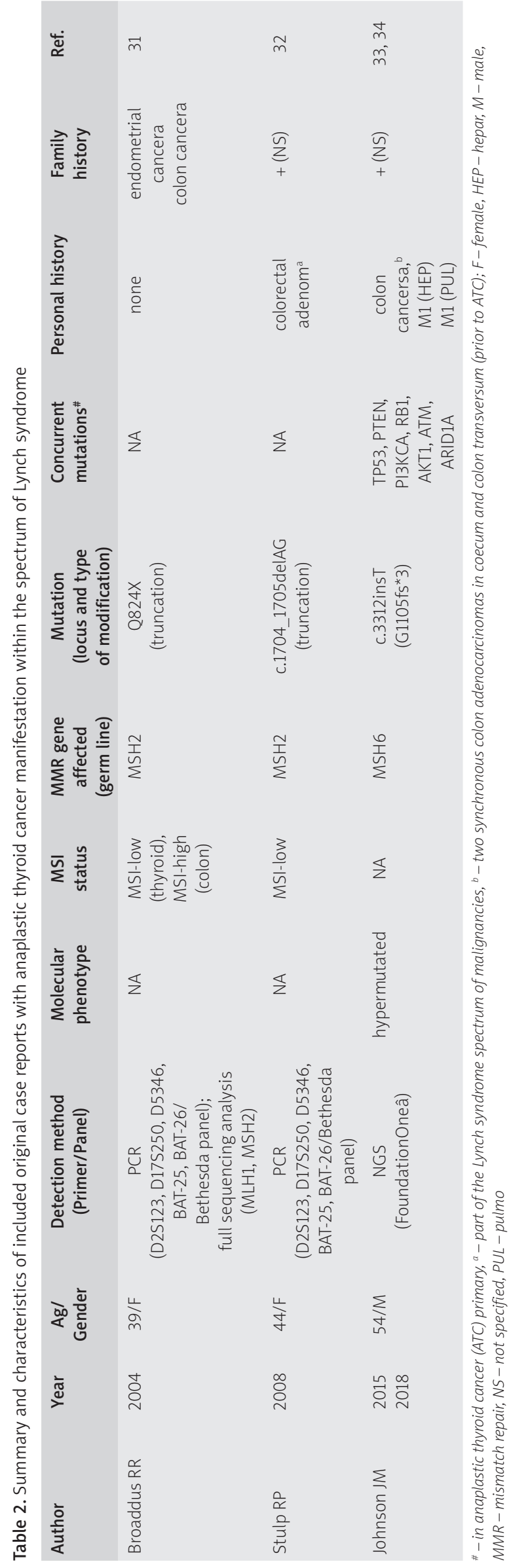

colorectal cancer syndrome. Carriers of germline mutations in the DNA MMR genes have a significantly increased risk of early cancer development, characteristically of the colon/ rectum, endometrial, skin, ovaries, kidneys, ureter, bladder, stomach, intestinal, pancreatobiliary system, and brain. In general, approximately $90 \%$ of DNA aberrations are found in the genes $\mathrm{MLH1}$ and $\mathrm{MSH}$, whereas barely $10 \%$ are noted in the genes MSH6 and PMS2 [30]. Interestingly, with regard to ATC, only 3 cases with documented germline mutations in the DNA MMR genes MSH2 and MSH6 have been reported so far [31-34] (Table 2). Of note, the 2 cases with MSH2 mutations presented with MSI-L status (in thyroid), whereas the MSI status for the case with MSH6 mutation was not given in the 2 published reports, but a hypermutated phenotype was protocolled (Table 2).

\section{Differences in testing panels}

In a larger scope, a review of the literature revealed a noteworthy study analysing MSI status with an adjusted reference panel, with special emphasis on thyroid cancer-specific loci (including microsatellites in the genes THRA1, thyroid hormone receptor $\alpha$, and TSHR, thyroid-stimulating hormone receptor; RET, p53), showing interesting clinicopathologic correlations: (I) MSI levels were as follows: THRA1 (36.5\%) > D2S123 (32.4\%) $>$ D11S912 (27.6\%) > D2S115 (22.4\%) > RET (18.8\%) > TSHR $(18.4 \%)>p 53(17.1 \%)>$ D2S399 $(14.3 \%)>$ BAT-26 (8.3\%); (II) MSI within the TSHR gene correlated with older patient age (> 70 years) and metastases to regional lymph nodes; (III) MSI in the dinucleotide marker D2S123 was detected in all FTCS (100\%), but only in a quarter of non-FTCs (25.3\%); and (IV) the mononucleotide marker BAT-26 displayed the lowest frequencies [35]. In contrast, the study by Genutis et al., including more than 480 tumour probes of different cancer types (PTC $[n=196]$, FTC $[n=156]$, PDTC $[n=18]$, $\operatorname{ATC}[n=51]$, MTC $[n=65]$ and oncocytic carcinoma [formerly Hürthle cell thyroid carcinoma; $n=3]$ ), analysed MSI status based on PCR and NGS, applying the Bethesda panel (BAT-25, BAT-26, D2S123, D17S250, D5S346). Remarkably, MSI was only detected in 4/156 FTCs (2.5\%) and was absent in all remaining 327 cases with other histomorphologies [25]. Of the 35 FTCs investigated by PCR herein, 2 cases presented instability at multiple microsatellites (case 1: BAT-26, D2S123; and case 2: BAT-25, BAT-26, D17S250) [25]. In this context, a third study should be mentioned: Santos et al. analysed 96 thyroid probes including PTCs (70 cases), FTCs (12 cases), follicular adenoma (FA, 7 cases), and normal tissue (7 cases) based on PCR, using the Bethesda panel plus 2 additional markers: BAT-40 and D11S912. The following astonishing frequencies of MSI status were given: 59/70 PTCS (84\%; MSI-H 64\%, MSI-L 36\%), FTCS 11/12 (92\%; MSI-H 82\%, MSI-L 18\%), and 6/14 FAs (43\%; MSI-L 100\%). MSI levels were as follows: D17S250 (37\%) $>\mathrm{D} 5 \mathrm{~S} 346(34 \%)>\mathrm{D} 2 \mathrm{~S} 123(19 \%)>\mathrm{D} 11 \mathrm{~S} 912(12 \%)>\mathrm{BAT}-$ $40(10 \%)>$ BAT-26 $(10 \%)>$ BAT-25 (2\%) [36]. These data also illustrate higher MSI frequencies in follicular-derived lesions. Comparable data were presented by Mitmaker et al. with MSI detected in 9/14 PTCs (64\%), and 10/16 FTCs (62.5\%), whereas 9/10 FAs were microsatellite-stable 
(MSS) or MSI-L [37]. However, PDTC and ATC cases were not the subject of the investigations of the latter 2 studies.

\section{Discussion}

Taking the facts of these studies together and earlier recommendations by the National Cancer Institute (NCI) into account, the detection of MSI in thyroid cancer and across other malignancies might require an adapted next-generation sequencing (NGS) reference panel and choice of different marker type in distinct histological subtypes [38]. This valuable approach is supported by data presented by Hause et al., who in an exome-based study investigated more than 200,000 microsatellite loci in 18 different cancer types ( $n=5930$ cases) (including PTC) and found cancer-specific loci that were more suitable in MSI detection [39]. The data of the studies listed above also indicate that a mononucleotide marker may not be appropriate in the setting of thyroid neoplasms [35-37], thus differing from its value in CRC and endometrial cancer (EC). Against this background, the major differences in the detection frequencies of MMR-d/MSI in variable thyroid neoplasms can be explained, with some studies illustrating a complete absence [28, 40]. Differences in detected prevalences might also result from varying molecular testing methods (e.g. targeted DNA sequencing [NGS], whole-exome sequencing [WES], whole-genome sequencing [WGS], polymerase chain reaction [PCR], capillary electrophoresis, and RNA-sequencing), specific sequencing platforms, and defined cut-offs (MSI-L versus MSI-H, number of tested microsatellite markers). Of note, optimal DNA sequencing coverage (or depth) is desirable, and better results have been achieved by NGS (IMPACT) compared to WES [15]. Beyond that, several computational tools have been introduced to screen the generated sequencing data (from NGS, WES, WGS) for MSI (e.g. MANTIS, mSINGS, MSISensor, MonoSeq, MOSAIC) [25, 39, 40], which may contribute to data standardization and identification of new MSI signatures in many cancer types.

Because the exact role of MSI in thyroid cancer initiation and dedifferentiation processes is still not well understood, there is controversy as to whether MMR-d/MSI represents a feature of early or late-stage disease. This can be explained by the fact that DNA MMR events or MSI are not only found in the later stages of PDTC and ATC, but also in the preceding FTC and PTC lesions, as well as in benign and tumour-like lesions such as FA and nodular hyperplasia/nodular goitre [14-17, 19, 22, 25, 35, 41-44]. Soares and Lazzereschi even documented 2 benign lesions with MSI-H status (1/13 nodular goitres plus 1/15 FAs and 2/16 FAs, respectively) $[19,41]$. Conversely, Hause et al. interpreted and discussed their MSI data along a continuum with MSI contributing to cancer progression [39]. This interpretation had already been made by Lazzereschi et al., who had found MSI (+) in lymph node metastases in 2 MSI (-) thyroid carcinomas (1 PTC, 1 HTC) and documented an increased prevalence of MSI (+) status at higher clinical stage, suggesting that MSI contributes to tumour expansion and evolution [19]. In line with this, an elegant single-case study by Paulsson et al. illustrated the coexistence of FTC, PDTC, and ATC, which were shown to be derived from 1 clone and presented an MSI signature in the PDTC and ATC parts, whereas FTC had borderline-MSI status [22]. Clonal evolution studies of Dong et al. led to the proposal of 2 ways of ATC progression: 2/5 ATCS with associated WDTC showed shared subclones, and 3/5 ATCS with associated WDTC had an independent clonal architecture [45].

Moreover, there are still insufficient knowledge and data concerning the actual prognostic value of MMR-d/MSI status with inconsistent, in part contradictory, analyses. In a large study conducted by Xu et al., including 360 ATCS, the aforementioned better prognostic outcome [16, 35] could not be reproduced [13]. Of note, the study by Onda et al. included only 2 ATC cases within a heterogenous cohort predominated by PTCs (85.6\%) [35]. A positive (nonsignificant) relationship between $\mathrm{MSI}+$ and prognosis was also reported by Hause et al., but the included TC cohort was not further specified as to histological subtypes [39]. An associated poor prognostic outcome of MSI+ tumours was discussed by Soares and Mitmaker, with both studies having exclusively analysed WDTCS (FTC, PTC) along with benign lesions, whilst PDTC and ATC cases were not the subject of the studies [37, 41].

Accordingly, these data cannot provide reliable conclusions or predictions because the prognostic impact of MMR-d/MSI may differ in each histological subtype and stage of disease. Differentiated assessment of the prognostic relevance of $M M R-d / M S I$ was already given in other malignancies (e.g. CRC, EC, gastric cancer, oesophageal cancer) [46-49], and staging parameters were shown to be relevant in part $[50,51]$. Future research and clinical investigations should also take prior treatments (chemotherapy, radiotherapy, etc.) into account because study results of other tumours, especially CRC and gastric cancer, tended to show a distinct association between MMR-d/ MSI status and chemotherapy response [46, 49, 51]. It further remains an open issue as to whether the association or increased frequencies of MMR-d/MSI in lesions with a follicular or follicular-derived morphology, as described by a few studies, represent robust results [22, 25, 35, 36].

Particularly noteworthy are the frequent co-mutations in the tumour suppressor gene TP53 in the setting of MMR gene modifications [14, 15, 17, 22]. Various genes of the DNA repair system display p53-corresponding sites, and it is suggested that the transcription factor p53 contributes to the transcriptional regulation of these genes, including MMR members such as MSH2 [22, 52]. Combined immunohistochemical studies of MMR/p53 might be of use and need to be explored because distinct expression patterns have been described in other tumour locations [53-55].

Besides somatic mutations in the MMR genes, inactivation of these repair mechanisms may also be caused by epigenetic modifications. In particular, promoter hypermethylation was reported for the DNA MMR gene MLH1. In a study by Guan et al., the prevalence of MLH1 methylation in 38 PTCs came to 21\% (8/38 PTCs), and an association with the BRAF V600E mutation as well as a positive nodal status was shown [56], thus demonstrating 
parallels to CRC $[57,58]$. In the study by Santos et al., MLH1 methylation frequencies were given as follows: 44\% PTCs (31/70 cases), 33\% FTCs (4/12 cases), and 64\% FAs (9/14 cases), and only a slight ("marginal") association with MSI status was postulated for the PTC cohort. However, no association with the BRAF V600E mutation was found [36]. Because there are currently very few data available in the area of FTC, PDTC, and ATC, further research and analyses are required. The same applies to $\mathrm{MSH} 2$ promotor hypermethylation as a result of EPCAM mutations, being reported in CRC [59].

The prior immunohistochemical evaluation of antibodies recognizing the 4 DNA MMR proteins MLH1, MSH2, MSH6, and PMS2, which function as heterodimers (e.g. MLH1-PMS2, syn. MutLa; MSH2-MSH6, syn. MutSa), may increasingly be of diagnostic value. At present, though, there are insufficient data available in thyroid cancer in general and in ATC in particular with respect to its correlation with NGS findings or interpretation of aberrant (weak, heterogenous, discordant) staining patterns, as reported in other tumours [60-63]. Further research efforts are required to evaluate the immunohistochemistry of MMR proteins as a first-line screening tool. Because MMR-d tumours of other locations and origins are shown to potentially respond to novel immunotherapies and may be associated with different prognostic outcome, corresponding patients need to be identified and risk-stratified, and emerging therapeutic options should be explored in the setting of aggressive ATC.

\section{Biomarker and target therapies for anaplastic thyroid cancer: limitations and perspective}

A comprehensive and precise understanding of the genetic and proteomic landscape underlying ATC in general and thorough histomorphological, immunohistochemical, and molecular analysis in individual cases are crucial for the identification and implementation of new anticancer treatment strategies. To explain all current approaches in detail would go beyond the framework of this review. However, a few interesting aspects and developments shall be mentioned.

A variety of single/combined-targeted therapies already exist or are evaluated within phase II clinical trials (compare ClinicalTrials.gov). These include tyrosine kinase inhibitors (TKIs), such as pazopanib, multikinase inhibitors (MKIs), such as sorafenib, sunitinib, and lenvatinib, and selective BRAF kinase inhibitors, such as vemurafenib, dabrafenib, or encorafenib combined with the MEK inhibitors such as trametinib, cobimetinib, binimetinib, or selumetinib [11, 12, 64-68]. The United States Food and Drug Administration (FDA) has already approved combined therapy with BRAF (dabrafenib)/MEK (trametinib) inhibitor in ATCS carrying BRAF V600-mutations [11, 69]. Moreover, combined therapies, e.g. fosbretabulin with paclitaxel/carboplatin or dual pathway blockade with MAPK and PI3K/ MTOR, have been evaluated, the latter with promising results in a single case study [70, 71]. One patient with a temporary response (18 months) to the mTOR inhibitor everolimus was also noted in a phase II study [72]. More- over, the results of studies evaluating the NTRK inhibitors larotrectinib and entrectinib were presented lately, with a substantial tumour response [73-75]. This has already led to FDA and the European Medicines Agency (EMA) approval for solid tumours with NTRK gene fusions. Furthermore, selpercatinib, a selective inhibitor of RET kinase, has been approved for RET fusion-positive thyroid cancer [76].

Over the past years, immuno-oncologic treatments, especially immune checkpoint inhibitors (ICls) (e.g. anti-programmed cell death-1 [PD-1], anti-programmed cell death-ligand 1 [PD-L1], and anti-cytotoxic T-lymphocyte-associated protein 4 [CTLA-4]) have revolutionized the field of anti-cancer therapies in many entities such as melanoma, non-small-cell lung carcinoma, Merkel cell carcinoma, renal cell carcinoma, classical Hodgkin lymphoma, squamous cell carcinoma of the head/neck, and urothelial carcinoma $[77,78]$. These drugs apply to signal-transducing cascades, which modulate immune system responses, with the PD-1/PD-L1 axis downregulating T cell-mediated innate and adaptive immune responses and preserving immune tolerance via inhibiting feedback mechanisms in the Ras-Raf-MEK-ERK and PI3K-AKT pathways [79]. Given the capacity of some tumour cells and immune cells (especially CD8+ T cells) of the cancer-specific microenvironment to express PD-L1, the PD-1/PD-L1 pathway represents an effective immunoediting and tumour escape mechanism by suppressing the effector phase of T-cell activation [80, 81].

Interestingly, some ATCs demonstrate relevant upregulation of inhibiting immune checkpoint regulators. Recent studies found the expression of the PD-L1 on cancer cells in up to $20-30 \%$ of analysed ATCs and slightly over $10 \%$ of PD-L1-positive intratumoural immune cells $[82,83]$. Another study by Cantara et al. (including 20 ATC patients) revealed more frequent expression of PD-L1 on tumour cells (70-90\% of ATCS), additionally showing tumour regression in a representative mouse model after anti-PD-L1 antibody application [84]. Also, in a murine ATC model, the combined therapy of anti-PD-L1 and a BRAF inhibitor was even more successful in tumour size reduction and increasing the number of tumour infiltrating immune cells [85]. Of note, a high PD-L1 amplification level in ATC was detected in about $5 \%$ within a large series of solid tumours including 177 cases of ATC [86]. The status of immune checkpoint blockade - as a single agent or in combination therapies with BRAF/MEK inhibitors - in ATCs/metastasized ATCS is presently the subject of more than 10 clinical phase I/II trials (as of March 2021), including the anti-PD-1/ PD-L1 antibodies pembrolizumab, nivolumab, atezolizumab, durvalumab, cemiplimab, and PDR001, among others (https://clinicaltrials.gov) [9, 10]. These trials were preceded by some very promising studies and single case reports that confirmed a favourable response, with one MMR-p case even demonstrating a complete response [82, 84, 87-89]. Encouraging results were also presented just recently after combined therapy with lenvatinib and pembrolizumab [90].

Nevertheless, the robustness of the immunopredictive value of PD-L1 to the immune checkpoint treatment response - as a solitary pan-tumour biomarker - has been questioned by several studies, in which this association 
could not be confirmed constantly [81, 91]. A synoptic report of different clinical trials analysing anti-PD1/PD-L1 therapy, including 1400 patients with solid tumours of varying entities, came to an overall response rate (ORR) of $48 \%$ in PD-L (+) tumours and, surprisingly, 15\% in PD-L1 $(-)$ tumours [92], making the necessity of a more reliable predictive biomarker clear. Accompanying difficulties arise from the challenging assessment of the PD-L1 immunohistochemistry, with distinct antibody applications for different tumour entities, variable cut-offs, defined scores, and interobserver variability in evaluating a true positive immunohistochemical reaction [93, 94], making meta-analytic research even more difficult.

The status of MMR deficiency or MSI was shown to be a further candidate serving as a predictive biomarker as regards the response to immune checkpoint blockade in several cancer subtypes [44, 95]. This is currently explained by the potential and ongoing generation of tumour-specific neoantigens (syn. mutation-associated neoantigens, MANAs) that results from high tumour mutational load, thereby inducing an immune response [44, 95-97]. With the extended approval of the immune checkpoint agent pembrolizumab (KEYTRUDA), a PD-1 receptor inhibitor, by the FDA in 2017 for all advanced staged, unresectable, or metastatic solid cancers in adult (and paediatric) patients with MMR-d or MSI-H status, independent of the tumour entity and primary tumour site (tissue/site-agnostic), oncologic treatment strategies and options have undergone a paradigm shift, and molecular testing of MMR-d/MSI has become increasingly important. The EMA, however, did not follow these recommendations. Instead, the tumourspecific immunopredictive responses were required thereupon to be evaluated precisely for each entity to receive treatment authorization, and in the meantime some progress has been made in various cancer types [44, 95, 98, 99].

Nevertheless, the expression pattern of PD-1/PD-L1 within this special molecular subtype of MMR-d/MSI signature in ATC is currently unknown. Further investigations are necessary to elucidate the impact of the PD-1/ PD-L1 axis blockade in this distinct subgroup of ATC: (I) with respect to MMR-p +/-TMB high, (II) in the context of MMR-d/MSI-H plus TMB high, (III) in MSI-L tumours, (IV) in MMR-d/MSI-H without TMB high, and (V) in MMR-d/ MSI-H without PD-1/PD-L1 expression but with a potential response, to name a few issues. The development of tumour type-specific, risk-stratified testing guidelines is also recommended.

It is finally worth mentioning that other potentially predictive biomarkers of response to $\mathrm{ICl}$ in thyroid (and non-thyroid) malignancies are currently being discussed and include TMB, APOBEC, MANAs, IFNg, TILS, and driver mutations, among others [100, 101]. Addressable structures and future therapies also include TAMs and cancer vaccines [102].

\section{Conclusions}

This study emphasized the small subgroup of ATCs with DNA MMR deficiency or MSI status. This subgroup obviously differs from conventional ATCs in a few clinicopathological parameters and is, in part, suggested to have better outcomes, although the tested cohorts were heterogenous and the prognostic value was not always applicable to ATC. However, given some promising results considering the response to immune checkpoint inhibitors in MMR-d/MSI-H tumours of other locations, ATCS also need to be analysed with a focus on the MSI signature, to identify patients with a distinct predictive/prognostic outcome, which can lead to adequate therapy. The results of immunotherapies in ATC, independent of MMR-d/MSI status, are currently being evaluated in numerous clinical trials, giving rise to the hope that improved treatment regimens will become available soon.

The diagnosis of ATC necessitates thorough differential diagnosis from other thyroid and non-thyroid malignancies as well as metastases to the thyroid. Tumour molecular profiling is fundamental in each ATC, not only for predictive and prognostic reasons, but also to elicit potential therapeutic options. Analysis of MSI status is strongly suggested and PD-L1 immunohistochemistry should be performed.

\section{Acknowledgements}

We thank Anett Grest, MA and Dipl.-Dok. Iris Nohsia from the scientific Campus Libraries of the Brandenburg Medical School Theodor Fontane for support in acquisition of full-text articles.

\section{The authors declare no conflict of interest.}

\section{References}

1. Wiseman SM, Loree TR, Rigual NR, et al. Anaplastic transformation of thyroid cancer: review of clinical, pathologic, and molecular evidence provides new insights into disease biology and future therapy. Head Neck 2003; 25: 662-670.

2. Molinaro E, Romei C, Biagini A, et al. Anaplastic thyroid carcinoma: from clinicopathology to genetics and advanced therapies. Nat Rev Endocrinol 2017; 13: 644-660.

3. Locati L, Cavalieri S, Dal Maso L, et al. Rare thyroid malignancies in Europe: data from the information network on rare cancers in Europe (RARECAREnet). Oral Oncol 2020; 108: 104766.

4. Janz TA, Neskey DM, Nguyen SA, et al. Is the incidence of anaplastic thyroid cancer increasing: a population based epidemiology study. World J Otorhinolaryngol Head Neck Surg 2019; 5: 34-40.

5. De Ridder M, van Dijkum EN, Engelsman A, et al. Anaplastic thyroid carcinoma: a nationwide cohort study on incidence, treatment and survival in the Netherlands over 3 decades. Eur J Endocrinol 2020; 183: 203-209.

6. Blomberg M, Feldt-Rasmussen U, Andersen KK, et al. Thyroid cancer in Denmark 1943-2008, before and after iodine supplementation. Int J Cancer 2012; 131: 2360-2366.

7. Smallridge RC, Copland JA. Anaplastic thyroid carcinoma: pathogenesis and emerging therapies. Clin Oncol 2010; 22: 486-497.

8. Kebebew E, Greenspan FS, Clark OH, et al. Anaplastic thyroid carcinoma: treatment outcome and prognostic factors. Cancer 2005; 103: 1330-1335.

9. Ma M, Lin B, Wang M, et al. Immunotherapy in anaplastic thyroid cancer. Am J Transl Res 2020; 12: 974-988.

10. D’Andréa G, Lassalle S, Guevara N, et al. From biomarkers to therapeutic targets: the promise of PD-L1 in thyroid autoimmunity and cancer. Theranostics 2021; 11: 1310-1325.

11. Subbiah V, Cabanillas ME, Kreitman RJ, et al. Dabrafenib and trametinib treatment in patients with locally advanced or metastat- 
ic BRAF V600-mutant anaplastic thyroid cancer. J Clin Oncol 2018; 36: 7-13.

12. Hyman DM, Puzanov I, Subbiah V, et al. Vemurafenib in multiple nonmelanoma cancers with BRAF V600 mutations. N Engl J Med 2015; 373: 726-736.

13. Xu B, Fuchs T, Dogan S, et al. Dissecting anaplastic thyroid carcinoma: a comprehensive clinical, histologic, immunophenotypic, and molecular study of 360 cases. Thyroid 2020; 30: 1505-1517.

14. Kunstman JW, Christofer Juhlin C, Goh G, et al. Characterization of the mutational landscape of anaplastic thyroid cancer via whole-exome sequencing. Hum Mol Genet 2015; 24: 2318-2329.

15. Landa I, Ibrahimpasic T, Boucai L, et al. Genomic and transcriptomic hallmarks of poorly differentiated and anaplastic thyroid cancers. J Clin Invest 2016; 126: 1052-1066.

16. Wong KS, Lorch JH, Alexander EK, et al. Clinicopathologic features of mismatch repair-deficient anaplastic thyroid carcinomas. Thy roid 2019; 29: 666-673.

17. Pozdeyev N, Gay LM, Sokol ES, et al. Genetic analysis of 779 advanced differentiated and anaplastic thyroid cancers. Clin Cancer Res 2018; 24: 3059-3068.

18. Ravi N, Yang M, Gretarsson S, et al. Identification of targetable lesions in anaplastic thyroid cancer by genome profiling. Cancers (Basel) 2019; 11: 402

19. Lazzereschi D, Palmirotta R, Ranieri A, et al. Microsatellite instability in thyroid tumours and tumour-like lesions. Br J Cancer 1999; 79: 340-345.

20. Landa I, Pozdeyev N, Korch C, et al. Comprehensive genetic characterization of human thyroid cancer cell lines: a validated panel for preclinical studies. Clin Cancer Res 2019; 25: 3141-3151.

21. Moher D, Liberati A, TetzlaffJ, et al. PRISMA 2009 Checklist - preferred reporting items for systematic reviews and meta-analyses. Ann Intern Med 2014; 18: 172-181.

22. Paulsson JO, Backman S, Wang N, et al. Whole-genome sequencing of synchronous thyroid carcinomas identifies aberrant DNA repair in thyroid cancer dedifferentiation. J Pathol 2020; 250: 183-194.

23. Nakagawa H, Lockman JC, Frankel WL, et al. Mismatch repair gene PMS2: disease-causing germline mutations are frequent in patients whose tumors stain negative for PMS2 protein, but paralogous genes obscure mutation detection and interpretation. Cancer Res 2004; 64: 4721-4727.

24. Yang J, Barletta JA. Anaplastic thyroid carcinoma. Semin Diagn Pathol 2020; 37: 248-256.

25. Genutis LK, Tomsic J, Bundschuh RA, et al. Microsatellite instability occurs in a subset of follicular thyroid cancers. Thyroid 2019; 29: 523-529.

26. Stenman A, Hellgren LS, Jatta K, et al. Metastatic anaplastic thyroid carcinoma in complete remission: morphological, molecular, and clinical work-up of a rare case. Endocr Pathol 2020; 31: 77-83.

27. Lai WA, Liu CY, Lin SY, et al. Characterization of driver mutations in anaplastic thyroid carcinoma identifies ras and pik3ca mutations as negative survival predictors. Cancers (Basel) 2020; 12: 1-13.

28. Vermiglio F, Schlumberger M, Lazar V, et al. Absence of microsatellite instability in thyroid carcinomas. Eur J Cancer 1995; 31: 128.

29. Dong W, Nicolson NG, Choi J, et al. Clonal evolution analysis of paired anaplastic and well-differentiated thyroid carcinomas reveals shared common ancestor. Genes Chromosom Cancer 2018 57: 645-652.

30. Lynch HT, de la Chapelle A. Hereditary colorectal cancer. N Engl Med 2003; 348: 919-932.

31. Broaddus RR, Lynch PM, Lu KH, et al. Unusual tumors associated with the hereditary nonpolyposis colorectal cancer syndrome. Mod Pathol 2004: 17: 981-989.

32. Stulp RP, Herkert JC, Karrenbeld A, et al. Thyroid cancer in a patient with a germline MSH2 mutation. Case report and review of the Lynch syndrome expanding tumour spectrum. Hered Cancer Clin Pract 2008; 6: 15-21.

33. Johnson JJM, Chen J, Dardi IK, et al. Molecular profile of synchronous metastatic colon cancer and anaplastic thyroid cancer in a patient with Lynch syndrome. J Clin Oncol 2015; 33: e22049-e22049.

34. Johnson JM, Chen J, Ali SM, et al. Molecular profiling of synchronous colon cancers and anaplastic thyroid cancer in a patient with Lynch syndrome. J Gastrointest Cancer 2018; 49: 203-206.
35. Onda M, Nakamura I, Suzuki S, et al. Microsatellite instability in thyroid cancer: Hot spots, clinicopathological implications, and prognostic significance. Clin Cancer Res 2001; 7: 3444-3449.

36. Santos JC, Bastos AU, Cerutti JM, et al. Correlation of MLH1 and MGMT expression and promoter methylation with genomic instability in patients with thyroid carcinoma. BMC Cancer 2013; 13: 79.

37. Mitmaker E, Alvarado C, Bégin LR, et al. Microsatellite instability in benign and malignant thyroid neoplasms. J Surg Res 2008; 150:40-48.

38. Boland CR, Thibodeau SN, Hamilton SR, et al. A National Cancer Institute Workshop on Microsatellite Instability for Cancer Detection and Familial Predisposition: development of international criteria for the determination of microsatellite instability in colorectal cancer. Cancer Res 1998 ; 58: 5248-5257.

39. Hause RJ, Pritchard CC, Shendure J, et al. Classification and characterization of microsatellite instability across 18 cancer types. Nat Med 2016; 22: 1342-1350

40. Bonneville R, Krook MA, Kautto EA, et al. Landscape of microsatellite instability across 39 cancer types. JCO Precis Oncol 2017; 1: 1-15.

41. Soares P, Dos Santos NR, Seruca R, et al. Benign and malignant thyroid lesions show instability at microsatellite loci. Eur J Cancer 1997: 33: 293-296.

42. Ruschenburg I, Vollheim B, Stachura J, et al. Analysis of DNA mismatch repair gene expression and mutations in thyroid tumours. Anticancer Res 2006; 26: 2107-2112.

43. Dobosz T, Łukienczuk T, Sa siadek $M$, et al. Microsatellite instability in thyroid papillary carcinoma and multinodular hyperplasia. Oncology 2000; 58: 305-310.

44. Le DT, Durham JN, Smith KN, et al. Mismatch repair deficiency predicts response of solid tumors to PD-1 blockade. Science 2017 357: 409-413.

45. Dong W, Nicolson NG, Choi J, et al. Clonal evolution analysis of paired anaplastic and well-differentiated thyroid carcinomas reveals shared common ancestor. Genes Chromosomes Cancer 2018; 57: 645-652.

46. Copija A, Waniczek D, Witkoś A, et al. Clinical significance and prognostic relevance of microsatellite instability in sporadic colorectal cancer patients. Int J Mol Sci 2017; 18: 107.

47. Nagle CM, O'Mara TA, Tan Y, et al. Endometrial cancer risk and survival by tumor MMR status. J Gynecol Oncol 2018; 29: 39.

48. Vrána D, Matzenauer M, Neoral Č, et al. From tumor immunology to immunotherapy in gastric and esophageal cancer. Int J Mol Sci 2019; 20: 13.

49. Haag GM, Czink E, Ahadova A, et al. Prognostic significance of microsatellite-instability in gastric and gastroesophageal junction cancer patients undergoing neoadjuvant chemotherapy. Int J Cancer 2019; 144: 1697-1703.

50. Mohan HM, Ryan E, Balasubramanian I, et al. Microsatellite instability is associated with reduced disease specific survival in stage III colon cancer. Eur J Surg Oncol 2016; 42: 1680-1686.

51. Smyth EC, Wotherspoon A, Peckitt C, et al. Mismatch repair deficiency, microsatellite instability, and survival. JAMA Oncol 2017; 3: 1197-1203.

52. Scherer SJ, Welter C, Zang KD, et al. Specific in vitro binding of p53 to the promoter region of the human mismatch repair gene hMSH2. Biochem Biophys Res Commun 1996; 221: 722-728.

53. Nyiraneza C, Jouret-Mourin A, Kartheuser A, et al. Distinctive patterns of $\mathrm{p} 53$ protein expression and microsatellite instability in human colorectal cancer. Hum Pathol 2011; 42: 1897-1910.

54. Mrózek A, Petrowsky H, Sturm I, et al. Combined p53/Bax mutation results in extremely poor prognosis in gastric carcinoma with low microsatellite instability. Cell Death Differ 2003; 10: 461-467.

55. Kunc M, Gabrych A, Rekawiecki B, et al. MLH1 promoter hypermethylation in uterine carcinosarcoma rarely coexists with TP53 mutation. Contemp Onkol 2019; 23: 202-207.

56. Guan H, Ji M, Hou P, et al. Hypermethylation of the DNA mismatch repair gene hMLH1 and its association with lymph node metastasis and T1799A BRAF mutation in patients with papillary thyroid cancer. Cancer 2008; 113: 247-255.

57. De Vogel S, Weijenberg MP, Herman JG, et al. MGMT and MLH1 promoter methylation versus APC, KRAS and BRAF gene muta- 
tions in colorectal cancer: Indications for distinct pathways and sequence of events. Ann Oncol 2009; 20: 1216-1222.

58. Weisenberger DJ, Siegmund KD, Campan M, et al. CpG island methylator phenotype underlies sporadic microsatellite instability and is tightly associated with BRAF mutation in colorectal cancer. Nat Genet 2006; 38: 787-793.

59. Ligtenberg MIL, Kuiper RP, Geurts van Kessel A, et al. EPCAM deletion carriers constitute a unique subgroup of Lynch syndrome patients. Fam Cancer 2013; 12: 169-174.

60. Joost P, Veurink N, Holck S, et al. Heterogenous mismatch-repair status in colorectal cancer. Diagn Pathol 2014; 9: 126.

61. Graham RP, Kerr SE, Butz ML, et al. Heterogenous MSH6 loss is a result of microsatellite instability within MSH6 and occurs in sporadic and hereditary colorectal and endometrial carcinomas. Am J Surg Pathol 2015; 39: 1370-1376.

62. Pai RK, Plesec TP, Abdul-Karim FW, et al. Abrupt loss of MLH1 and PMS2 expression in endometrial carcinoma. Am I Surg Pathol 2015; 39: 993-999.

63. Kato A, Sato N, Sugawara T, et al. Isolated loss of PMS2 immunohistochemical expression is frequently caused by heterogenous MLH1 promoter hypermethylation in lynch syndrome screening for endometrial cancer patients. Am J Surg Pathol 2016; 40: 770-776.

64. Bible KC, Suman VJ, Menefee ME, et al. A multiinstitutional phase 2 trial of pazopanib monotherapy in advanced anaplastic thyroid cancer. J Clin Endocrinol Metab 2012; 97: 3179-3184.

65. Capdevila J, Iglesias L, Halperin I, et al. Sorafenib in metastatic thyroid cancer. Endocr Relat Cancer 2012; 19: 209-216.

66. Ravaud A, de la Fouchardière C, Caron P, et al. A multicenter phase II study of sunitinib in patients with locally advanced or metastatic differentiated, anaplastic or medullary thyroid carcinomas: mature data from the THYSU study. Eur I Cancer 2017; 76: 110-117.

67. Tahara M, Kiyota N, Yamazaki T, et al. Lenvatinib for anaplastic thyroid cancer. Front Oncol 2017; 7: 139-144.

68. Cabanillas ME, Ryder M, Jimenez C. Targeted therapy for advanced thyroid cancer: kinase inhibitors and beyond. Endocr Rev 2019; 40: 1573-1604.

69. Wang JR, Zafereo ME, Dadu R, et al. Complete surgical resection following neoadjuvant dabrafenib plus trametinib in BRAFV600E-mutated anaplastic thyroid carcinoma. Thyroid 2019 29: 1036-1043.

70. Sosa JA, Elisei R, Jarzab B, et al. Randomized safety and efficacy study of fosbretabulin with paclitaxel/carboplatin against anaplastic thyroid carcinoma. Thyroid 2014; 24: 232-240.

71. Gibson WJ, Ruan DT, Paulson VA, et al. Genomic heterogeneity and exceptional response to dual pathway inhibition in anaplas tic thyroid cancer. Clin Cancer Res 2017; 23: 2367-2373.

72. Wagle N, Grabiner BC, Van Allen EM, et al. Response and acquired resistance to everolimus in anaplastic thyroid cancer. N Engl J Med 2014; 371: 1426-1433.

73. Drilon A, Laetsch TW, Kummar S, et al. Efficacy of larotrectinib in TRK fusion-positive cancers in adults and children. N Engl J Med 2018; 378: 731-739.

74. Liu D, Offin M, Harnicar S, et al. Entrectinib: an orally available, selective tyrosine kinase inhibitor for the treatment of NTRK, ROS1, and ALK fusion-positive solid tumors. Ther Clin Risk Manag 2018; 14: 1247-1252.

75. Cabanillas ME, Zafereo M, Williams MD, et al. Recent advanc es and emerging therapies in anaplastic thyroid carcinoma. F1000Research 2018; 7: F1000 Faculty Rev-87.

76. Della Corte CM, Morgillo F. Rethinking treatment for RET-altered lung and thyroid cancers: selpercatinib approval by the EMA. ESMO Open 2021; 6: 100041

77. Alsaab HO, Sau S, Alzhrani R, et al. PD-1 and PD-L1 checkpoint sig naling inhibition for cancer immunotherapy: mechanism, combinations, and clinical outcome. Front Pharmacol 2017; 8: 561.

78. Jiang Y, Chen M, Nie H, et al. PD-1 and PD-L1 in cancer immunotherapy: clinical implications and future considerations. Hum Vaccines Immunother 2019; 15: 1111-1122.

79. Patsoukis N, Brown J, Petkova V, et al. Selective effects of PD-1 on Akt and ras pathways regulate molecular components of the cell cycle and inhibit T cell proliferation. Sci Signal 2012; 5: ra46.

80. Topalian SL, Hodi FS, Brahmer JR, et al. Safety, activity, and immune correlates of anti-PD-1 antibody in cancer. N Engl J Med 2012; 366: 2443-2454.
81. Postow MA, Callahan MK, Wolchok JD. Immune checkpoint blockade in cancer therapy. J Clin Oncol 2015; 33: 1974-1982.

82. Zwaenepoel K, Jacobs J, de Meulenaere A, et al. CD70 and PD-L1 in anaplastic thyroid cancer - promising targets for immunotherapy. Histopathology 2017 ; 71: 357-365.

83. Ahn S, Kim TH, Kim SW, et al. Comprehensive screening for PD-L1 expression in thyroid cancer. Endocr Relat Cancer 2017; 24: 97-106.

84. Cantara S, Bertelli E, Occhini R, et al. Blockade of the programmed death ligand 1 (PD-L1) as potential therapy for anaplastic thyroid cancer. Endocrine 2019; 64: 122-129.

85. Brauner E, Gunda V, Borre PV, et al. Combining BRAF inhibitor and anti PD-L1 antibody dramatically improves tumor regression and anti tumor immunity in an immunocompetent murine model of anaplastic thyroid cancer. Oncotarget 2016; 7: 17194-17211.

86. Goodman AM, Piccioni D, Kato S, et al. Prevalence of PDL1 amplification and preliminary response to immune checkpoint blockade in solid tumors. JAMA Oncol 2018; 4: 1237-1244.

87. Spalart V, Legius B, Segers K, et al. Dramatic response to first line single agent pembrolizumab in anaplastic thyroid carcinoma. Case Rep Endocrinol 2019; 2019.

88. Stenman A, Hellgren LS, Jatta K, et al. Metastatic anaplastic thyroid carcinoma in complete remission: morphological, molecular, and clinical work-up of a rare case. Endocr Pathol 2020; 31: 77-83.

89. Capdevila J, Wirth LJ, Ernst T, et al. PD-1 blockade in anaplastic thyroid carcinoma. J Clin Oncol 2020; 38: 2620-2627.

90. Dierks C, Seufert J, Aumann K, et al. The lenvatinib and pembrolizumab combination is an effective treatment option for anaplastic and poorly differentiated thyroid carcinoma. Thyroid 2021; 31.

91. Yi M, Jiao D, Xu H, et al. Biomarkers for predicting efficacy of PD-1/ PD-L1 inhibitors. Mol Cancer 2018; 17: 129.

92. Sunshine J, Taube JM. PD-1/PD-L1 inhibitors. Curr Opin Pharmacol 2015; 23: 32-38.

93. Patel SP, Kurzrock R. PD-L1 expression as a predictive biomarker in cancer immunotherapy. Mol Cancer Ther 2015; 14: 847-856.

94. Schildhaus HU. Predictive value of PD-L1 diagnostics. Pathologe 2018; 39: 498-519.

95. Le DT, Uram JN, Wang H, et al. PD-1 Blockade in Tumors with Mismatch-Repair Deficiency. N Engl J Med 2015; 372: 2509-2520.

96. Samstein RM, Lee CH, Shoushtari AN, et al. Tumor mutational load predicts survival after immunotherapy across multiple cancer types. Nat Genet 2019; 51: 202-206.

97. Chan TA, Yarchoan M, Jaffee E, et al. Development of tumor mutation burden as an immunotherapy biomarker: Utility for the oncology clinic. Ann Oncol 2019; 30: 44-56.

98. Abida W, Cheng ML, Armenia J, et al. Analysis of the Prevalence of Microsatellite Instability in Prostate Cancer and Response to Immune Checkpoint Blockade. JAMA Oncol 2019; 5: 471-478.

99. Hashimoto T, Kurokawa Y, Takahashi T, et al. Predictive value of MLH1 and PD-L1 expression for prognosis and response to preoperative chemotherapy in gastric cancer. Gastric Cancer 2019; 22: 785-792.

100. Faden DL, Ding F, Lin Y, et al. APOBEC mutagenesis is tightly linked to the immune landscape and immunotherapy biomarkers in head and neck squamous cell carcinoma. Oral Oncol 2019; 96: 140-147.

101. Wang S, Jia M, He Z, et al. APOBEC3B and APOBEC mutational signature as potential predictive markers for immunotherapy response in non-small cell lung cancer. Oncogene 2018; 37: 3924-3936.

102. Ma M, Lin B, Wang M, et al. Immunotherapy in anaplastic thyroid cancer. Am J Transl Res. 2020; 12: 974-988.

\section{Address for correspondence}

Piotr Czapiewski, MD

Institute of Pathology

Dessau Medical Centre

Auenweg 38

06847 Dessau, Germany

e-mail: p.czapiewski@klinikum-dessau.de

Submitted: 25.09 .2021

Accepted: 25.09 .2021 